\title{
An intelligent loader-digger heat balance system based on simulation and wireless sensor networks
}

\author{
Lei Li ${ }^{*}$, Xi Chen ${ }^{1}$, Di Liu' ${ }^{1}$ Hong-gen Zhou ${ }^{1}$ and Haiyan $\mathrm{Wu}^{2}$
}

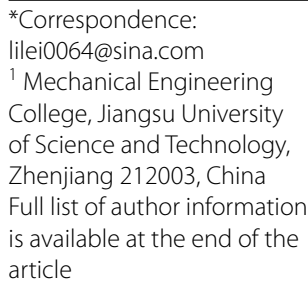

\begin{abstract}
High temperature of the radiator group is harmful to the power system and hydraulic system. In order to improve the heat dissipation performance of the loader, the flow field characteristics of the cooling chamber are analyzed by simulation and heat balance test. Firstly, the mathematical model of heat flow is established. Secondly, the flow field in the cooling chamber under different speeds is simulated based on CFX. And then the influence of fan position and internal flow field distribution on radiator performance is studied. Through the simulation of four different distances, it is concluded that the optimal distance between cooling fan and radiator is $76 \mathrm{~mm}$. Finally, the heat balance test system based on wireless sensor network is established, and the simulation results are verified. The test results show that the heat dissipation performance of the whole machine meets the requirements after optimization. In this paper, the proposed scheme can dynamically adjust the heat dissipation performance and make the loader excavator in an efficient and energy-saving operation state.
\end{abstract}

Keywords: Loader-digger, CFX, Flow performance, Heat dissipation performance, Temperature testing system

\section{Introduction}

With the development of information technology, the Internet of things has become a major event in our daily life of the next generation. It is defined as a global network with the ability to work with a self-configuring infrastructure to deploy various types of sensors to collect different environmental information, such as temperature, humidity, light intensity, voltage, and current. In addition, it can monitor the specific state and realize the psychological information through the comprehensive analysis of the environment. Sensor nodes have limited computer space power and some communication capabilities, and can transmit relevant information after sensing the network computing. It is commonly used in traditional areas such as military defense, biomedical and environmental monitoring, and plays an important role in emerging areas such as smart home, urban transportation.

The loader-digger is a multi-functional construction machinery product with both loading and excavation functions. It is widely used in industrial and agricultural

OThe Author(s) 2020. Open Access This article is licensed under a Creative Commons Attribution 4.0 International License, which permits use, sharing, adaptation, distribution and reproduction in any medium or format, as long as you give appropriate credit to the original author(s) and the source, provide a link to the Creative Commons licence, and indicate if changes were made. The images or other third party material in this article are included in the article's Creative Commons licence, unless indicated otherwise in a credit line to the material. If material is not included in the article's Creative Commons licence and your intended use is not permitted by statutory regulation or exceeds the permitted use, you will need to obtain permission directly from the copyright holder. To view a copy of this licence, visit http:// creativecommons.org/licenses/by/4.0/. 
production and defense industries. The heat load of the loader-digger heat removal system refers to the heat of the engine system, transmission system, and hydraulic system radiator. Most of this heat is dissipated through the convection heat exchanger of the radiator. The heat of the engine, transmission and hydraulic system is, respectively, mainly distributed by the engine water radiator, air-air cooler, torque converter oil radiator and hydraulic oil radiator. Because the working environment of the loader-digger is complex and diverse, the heat balance performance of the whole machine is required to be high. The engine, loading system, excavation system, and traveling system all need to be below the rated temperature in order to carry out various operations safely and efficiently.

There are three methods for evaluating the heat dissipation performance of construction machinery: First, thermal balance vehicle test. This method is the most direct and simple, but it has long cycle, high cost and poor repeatability. Second, it is a bench simulation test. This method is more accurate, with a higher degree of quantification, but it also costs a lot. The test equipment is extremely demanding. The third is the simulation technology. Through the establishment of the target product theory and simulation model, the use of simulation software for calculation and analysis, combined with physical experiments for comprehensive evaluation, this method can save a lot of time and manpower and resources, has become the most effective means of evaluating the thermal performance of complex machinery products one.

Wang et al. [1] studied the changes of the flow field and temperature field in the cabin, using a combination of numerical simulation and experimentation. Chang et al. [2] used a heavy-duty diesel engine for loaders. The heat balance system performed onedimensional simulation analysis. The error between the simulation results and the test data was less than $10 \%$. Sortor [3] compares the effects of the cooling module mass flow, fan energy consumption and fan efficiency characteristics on the heat balance system based on two different turbulent computational fluid dynamics (CFD) models. Zhang and Wang [4] simulated the airflow in the engine compartment of multipurpose vehicle (MPV) based on Hypermesh and Fluent software. The heat balance of the engine compartment of the car is analyzed to solve the problem of excessively high engine water temperature. Khaled et al. [5] combined the flow network modeling (FNM) fluid grid model with the CFD computational fluid dynamics method. The relationship was studied between the position layout of the electric and mechanical components in the power cabin and the influence of the temperature field. Khaled [6] optimized the hood cooling module and proposed a method to control the positioning of the cooling module using the engine's energy demand, which reduced the heat transferred from the engine to the pump and the compression chamber, achieving the goal of high efficiency and energy saving. Datnodaran and Rahman [7] was based on the FLUENT software platform to study the internal flow field and temperature field of the automobile power cabin. The simulation results were verified by wind tunnel test. The feasibility of solving the heat problem of the power cabin through the CFD method was verified. Kambiz and Beigmoradi [8] analyzed the impact of the different shape of the inlet grid on the flow field in the power cabin. A special front grille was constructed with the guide plate and the baffle plate. The inlet flow rate of the power cabin was increased by $10 \%$, and the heat dissipation efficiency of the radiator was improved. Saha and Acharya [9] studied the 
heat dissipation system of heavy load truck. The heat dissipation process of its power cabin was analyzed. A comprehensive evaluation of the heat dissipation effect of the power cabin was established, which was helpful to the early design of the heat dissipation system of heavy load truck. Zhang et al. [10] designed the wind pipe between the radiator and the inlet grille, which improves the uniformity of the airflow of the radiator. $\mathrm{Qu}$ et al. [11] analyzed the air flow field inside the power room of liquefied petroleum gas (LPG) vehicles and found that the air velocity and the range of thermal fluid have a certain influence on the cooling effect of the heat balance system. Liu et al. [12] studied the relationship between the cooling fan speed and the heat dissipation performance of the radiator in order to find out the performance law of the engine heat balance system of the dual-fan engineering vehicle. Dong and Liu [13] proposed a method of heat flux coupling topology optimization based on density, which transformed the optimization design problem into a multi-objective optimization problem including heat resistance, energy dissipation and pressure drop. Wang et al. [14] combined a full automatic multi-objective optimization with coupled heat transfer calculation to obtain the optimal cooling arrangement of transonic high-pressure guide vanes. Lu et al. [15] carried out experimental research and parameter analysis on hhd-pvt-hp system, and the results show that the change of wind speed has a significant impact on the cooling performance of PVT module. Wang et al. [16] studied the effects of ventilation, ampacity and cable position on the temperature of the power room cable through the scale model and CFD simulation. Li et al. [17] studied the airflow and temperature fields of EPSC based on CFD and experiments. By analyzing the relationship between the intake parameters and the environment in the EPSC, a scheme that helps optimize the design of the powerhouse is obtained.

In order to solve the problem of overheating and residual heat accumulation in the loader-digger, the CFX is used to simulate and analyze the air flow of the heat balance system under the running condition. The distribution of the internal flow field under different speeds conditions is analyzed. The influence of the relative position of the fan and the radiator on the internal flow field is studied. And, the hood structure is optimized. Then, the cooling efficiency of the system under the running condition is improved.

The main contributions of this paper include:

1 Establish a mathematical model for the circulation in the radiator cabin, and mesh and set the boundary conditions for the simulation model.

2 The simulation on the flow performance at different vehicle speeds and the relative position of the cooling fan and radiator is based on CFX, an optimization plan for the hood of the radiator cabin is proposed.

3 The heat balance test system based on wireless sensor network is established. Using sensors to obtain a large number of state evaluation data, the heat balance system can be dynamically adjusted to achieve the best state.

The remainder of this paper is organized as follows. In Sect. 2, we establish the mathematical model of turbulent fields. Section 3 describes the numerical method. In Sect. 4, we analyze the simulation of the heat dissipation system of the excavator loader. We set up a heat balance test system based on wireless sensor network to evaluate the performance of 
the excavator loader system in Sect. 5. Finally, Sect. 6 concludes this paper and points out future research directions.

\section{Mathematical models}

The flow field in the bilge chamber of the loader-digger contains both flow transfer problems. Due to the low gas flow velocity in the chiller cabin, it can be considered as a noncompressible fluid calculation. The continuous equations of incompressible fluids and the Reynolds Navier-Stokes equations can be expressed as follows:

$$
\begin{aligned}
& \frac{\partial \rho}{\partial t}+\frac{\partial \rho U_{i}}{\partial x_{i}}=0 \\
& \frac{\partial \rho U_{i}}{\partial t}+\frac{\partial \rho U_{i} U_{j}}{\partial x_{j}}=-\frac{\partial p}{\partial x_{i}}+\frac{\partial}{\partial x_{i j}}\left[\left(\mu+\mu_{T}\right)\left(\frac{\partial U_{i}}{\partial x_{j}}+\frac{\partial U_{j}}{\partial x_{i}}\right)\right]
\end{aligned}
$$

where $U_{i}$ represents the $i$ component of velocity, $t$ represents time, $x_{i}$ represents Cartesian coordinate, $\rho$ represents fluid density, $p$ represents dynamic pressure, $\mu$ represents viscosity, $\mu_{T}$ represents eddy viscosity.

In fluid mechanics, the $k-\varepsilon$ double equation model is often used to describe the turbulent flow field. The commonly used $k-\varepsilon$ double equation models include Standard $k-\varepsilon$ turbulence model, Realizable $k-\varepsilon$ turbulence model and RNG $k-\varepsilon$ turbulence model. Due to the complexity of the flow field in the bilge chamber of the loader-digger, the Realizable $k-\varepsilon$ turbulence model adds a new equation to calculate the dissipation rate, which is even more significant for swirling flow, boundary laminar flow, flow separation and complex two-phase flow. Accurate, so the Realizable $k-\varepsilon$ turbulence model is used to describe the turbulent flow field in the cooling chamber. The turbulence model is as follows:

$$
\begin{aligned}
& \frac{\partial(\rho k)}{\partial t}+\frac{\partial\left(\rho k u_{i}\right)}{\partial x_{i}}=\frac{\partial}{\partial x_{j}}\left[\left(\mu+\frac{\mu_{t}}{\sigma_{k}}\right) \frac{\partial k}{\partial x_{j}}\right]+G_{K}+G_{b}-\rho \varepsilon-Y_{M}+S_{k} \\
& \frac{\partial(\rho \varepsilon)}{\partial t}+\frac{\partial\left(\rho \varepsilon u_{i}\right)}{\partial x_{i}}=\frac{\partial}{\partial x_{j}}\left[\left(\mu+\frac{\mu_{t}}{\sigma_{\varepsilon}}\right) \frac{\partial \varepsilon}{\partial x_{j}}\right]+\rho C_{1} S \varepsilon-\rho C_{2} \frac{\varepsilon^{2}}{k+\sqrt{v \varepsilon}}+C_{1 \varepsilon} \frac{\varepsilon}{k} G_{3 \varepsilon} G_{b}+S_{\varepsilon} \\
& C_{1}=\max \left[0.43, \frac{\eta}{\eta+5}\right], \eta=S \frac{k}{\varepsilon}, S=\sqrt{2 S_{i j} S_{i j}}, \mu_{t}=\rho C_{\mu} \frac{k^{2}}{\varepsilon}
\end{aligned}
$$

where $\rho$ represents fluid density; $x_{i}$ and $x_{j}$ represent each coordinate component; $\mu$ represents molecular viscosity coefficient; $\mu_{t}$ represents turbulent viscosity coefficient; $\sigma_{k}$ and $\sigma_{\varepsilon}$ represent the turbulent Prandtl number of turbulent kinetic energy $k$ and dissipation rate $\varepsilon ; G_{K}$ represents turbulent kinetic energy produced by mean velocity gradient; $G_{b}$ represents the turbulent kinetic energy produced by the impact of buoyancy; $Y_{M}$ represents the influence of the dynamic expansion of the compressible iliac artery on the total dissipation rate; $S_{\varepsilon}$ and $S_{k}$ represent the user-defined source terms for the model; $C_{2}, C_{1 \varepsilon}, C_{3 \varepsilon}$ are constant. 
Table 1 Radiator parameters

\begin{tabular}{lll}
\hline Name & Length * Width * Height $(\mathbf{m m})$ & Temperature requirements \\
\hline Transmission oil radiator & $635 * 70 * 225$ & $\mathrm{TOC} \leq 100^{\circ} \mathrm{C}$ \\
Hydraulic oil radiator & $635 * 70 * 490$ & $\mathrm{HOC} \leq 100^{\circ} \mathrm{C}$ \\
Cooling radiator & $635 * 110 * 650$ & $\mathrm{RAD} \leq 105^{\circ} \mathrm{C}$ \\
Air-air cooler & $710 * 110 * 130$ & $\mathrm{CAC} \leq$ ring temperature $+30^{\circ} \mathrm{C}$ \\
\hline
\end{tabular}

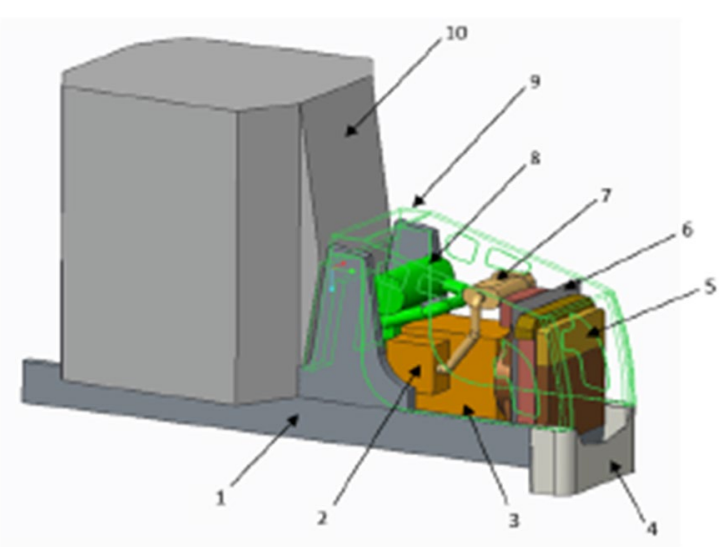

1. Rack 2. Turbo charger 3. Engine 4. Counter weight 5. Radiator Set

6. Air Hood 7. Air Filter 8. Muffler 9. Hood 10. Cab

Fig. 1 Radiating cabin simplified model

\section{Description of the numerical method}

\subsection{Mesh generation}

In order to fully analyze the cooling capacity of the loader-digger, the physical model of the loader-digger is properly simplified, retaining the main heat source (engine, turbocharger and muffler) and components which can significantly affect the direction of air flow (air cleaner, air hood, intake and exhaust pipe, frame, hood, counterweight and $\mathrm{cab})$. The simulation condition is the running condition of the sports car, and the loading end is at the lowest position. At this time, the loading end has little influence on the flow field in the heat-dissipating cabin, so the interference of the loading end is not considered. The radiators are all air-cooled, including transmission oil radiators, hydraulic oil radiators, engine cooling radiators, and air-air coolers. Their structure is a plate-fin structure. The dimensions and temperature requirements of each radiator are shown in Table 1. As shown, the fan is an eight-blade suction type with a maximum blade speed of $2200 \mathrm{rad} / \mathrm{s}$.

According to the relevant technical data, the simulation model of the radiating cabin of a certain type of loader-digger is shown in Fig. 1. Main components include radiators, fans, engines, turbochargers, mufflers, air cleaners, wind deflectors, intake and exhaust pipes, racks, hoods, counterweights, and cabs.

In order to analyze the internal characteristics of the radiating cabin in the working conditions of the excavating loader, a virtual wind tunnel simulation model is established. Since the front air intake of the vehicle is affected by the external flow field, a 
calculation domain suitable for the external flow field is adopted. The size of the external flow field is 10 times the length of the vehicle, 5 times the width of the vehicle and 3 times the height of the vehicle. The size of the calculation domain is: the distance between the entrance and the front of the loader-digger is $10 \mathrm{~m}$. The distance between the exit and the back of the loader-digger is $20 \mathrm{~m}$. The distance between the upper wall and the top of the cab is $10 \mathrm{~m}$. And, the distance between the two side walls and the sides of the excavator loader is $5 \mathrm{~m}$.

Due to the complex structure of the heat-dissipating cabin, a tetrahedral grid with good adaptability is selected for the heat-dissipation cabin and the periphery of the car body. The structure of the radiator group is regular and a hexahedral grid is adopted. The flow field on the surface of the heat-dissipating cabin changes drastically. And, the surface of the heat-dissipating cabin is refined. The results of meshing are shown in Fig. 2. The total number of meshes in all computational domains is approximately 7.05 million.

\subsection{Boundary conditions}

For the change of the flow field and temperature field inside the radiating cabin, the following simplifications and assumptions are made in the numerical simulation. A threedimensional steady-state flow field is adopted, and the inlet wind speed is uniform. The air flow rate in the heat-dissipating cabin is low and can be considered as incompressible Newtonian fluid, turbulence model using Realizable $k-\varepsilon$ model. The effects of thermal radiation and gravity field are ignored. The convection with the outside air is ignored. The loader-digger is full load, and the environment is the worst case.

The boundary conditions are set as follows: The entrance is set to speed entrance, the speeds are, respectively, $0 \mathrm{~km} / \mathrm{h}, 6.8 \mathrm{~km} / \mathrm{h}, 11.1 \mathrm{~km} / \mathrm{h}, 23.8 \mathrm{~km} / \mathrm{h}, 42.5 \mathrm{~km} / \mathrm{h}$. The exit is set to free flow exit, the pressure is $0 \mathrm{~Pa}$. The fan speed is $2200 \mathrm{rpm}$. The ambient temperature is $45^{\circ} \mathrm{C}$. The radiator is set to a porous medium and the material is aluminum.

\section{Simulation of heat dissipation system based on CFX}

\subsection{Prediction of flow performance at different speeds}

The change of vehicle speed affects the flow rate of the air inlet of the hood and the rotation speed of the cooling fan, thereby changing the distribution of the internal flow field of the radiator cabin. According to the speed of each gear, five operating conditions of $0,6.8,11.1,23.8$, and $42.5 \mathrm{~km} / \mathrm{h}$ were selected, and a comparative

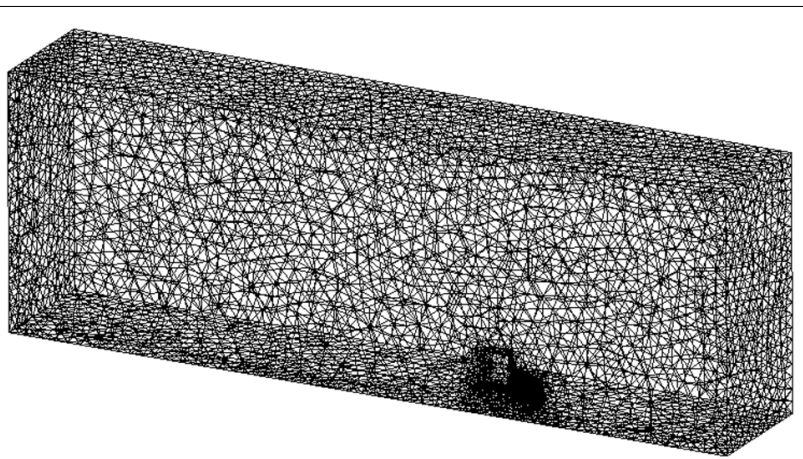

Fig. 2 Compute domain meshing 


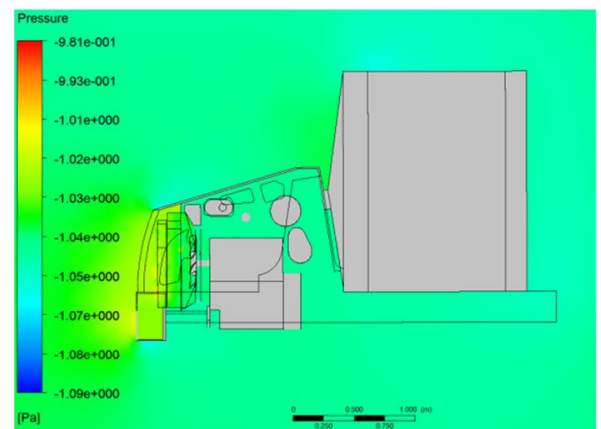

a Section pressure map

Fig. 3 Section pressure and speed diagram at idle $(x=0)$

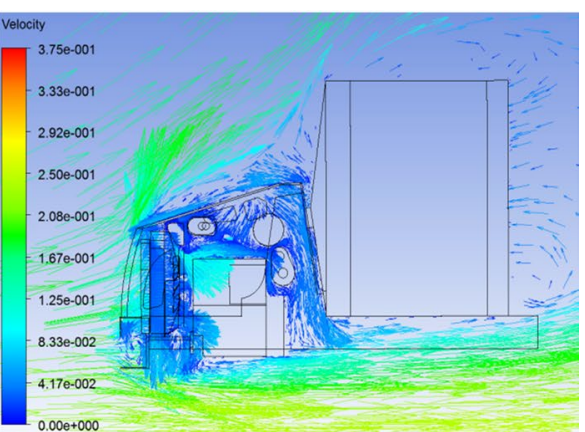

b Speed vector

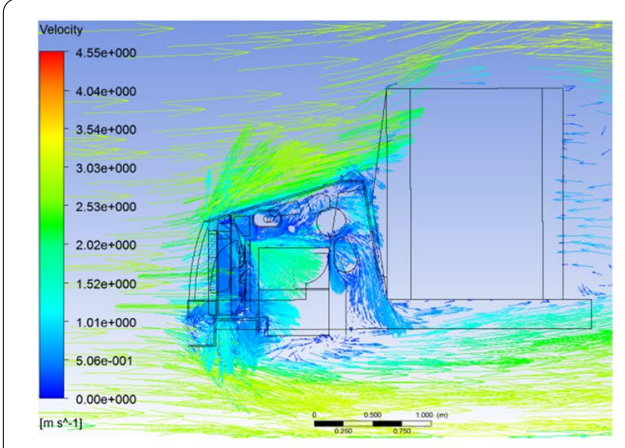

a $11.1 \mathrm{~km} / \mathrm{h}$

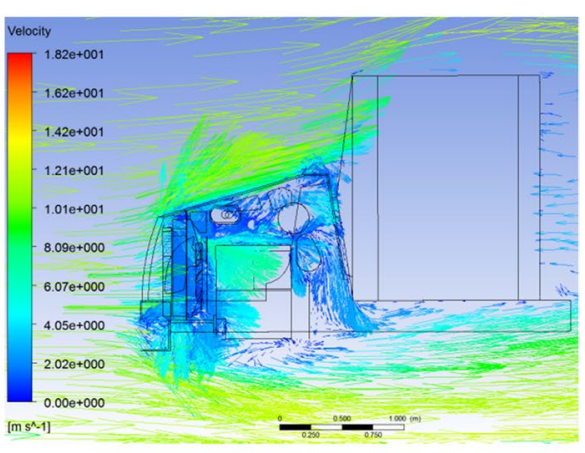

b $42.5 \mathrm{~km} / \mathrm{h}$

Fig. 4 Cross-sectional speed vector at different speeds $(x=0)$

analysis of the air flow in the radiator cabin and the pressure cloud diagram under the same vehicle environment was conducted.

Figure 3a is a vertical cross-sectional pressure cloud diagram at the center of the bilge chamber when the loader-digger is idling. Due to the suction effect of the fan, a large low-pressure zone is generated near the radiator group at the front of the fan. A high-pressure zone is formed near the engine at the rear of the fan, and at the entrance of the radiator compartment and the air intake at the bottom of the engine. The pressure is also higher, so the difference in pressure causes the outside air to flow into the radiating cabin. Figure $3 \mathrm{~b}$ shows the speed vector diagram. The air entering from the air inlet is directly sucked by the fan, flows through the radiator group, passes through the air hood and the fan, and then from the engine, the air intake system and the muffler. The surface of the components and parts flows through them, and finally they are discharged from the bottom of the heat-dissipating chamber and the air outlets on both sides of the hood to the heat-dissipating chamber. There is no serious backflow near the engine, and the overall flow of the cooling chamber in the cross section is smooth.

According to Figs. 3 and 4, when the vehicle speed is low, the fan pressure rise and the air hood influence the intake air volume very much. As the vehicle speed 


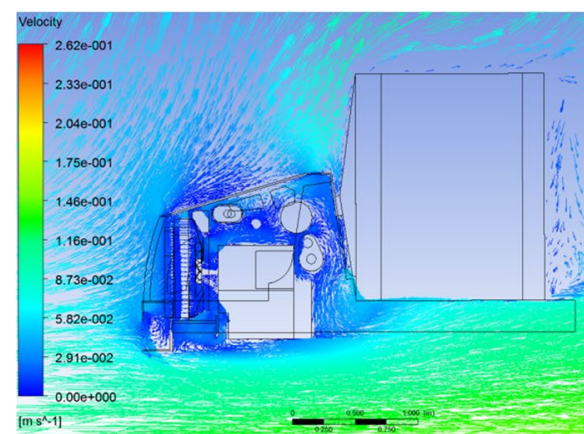

a Option 1

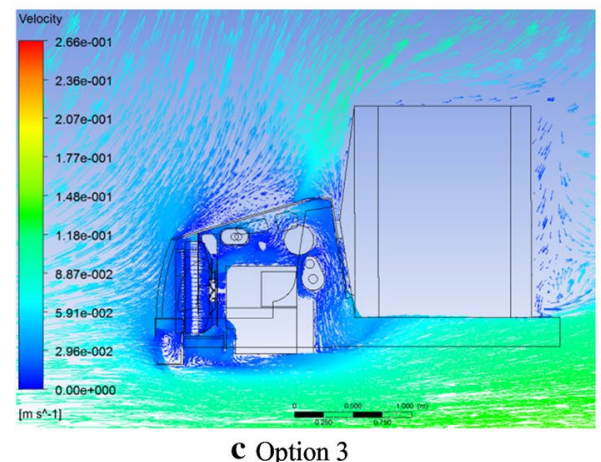

c Option 3

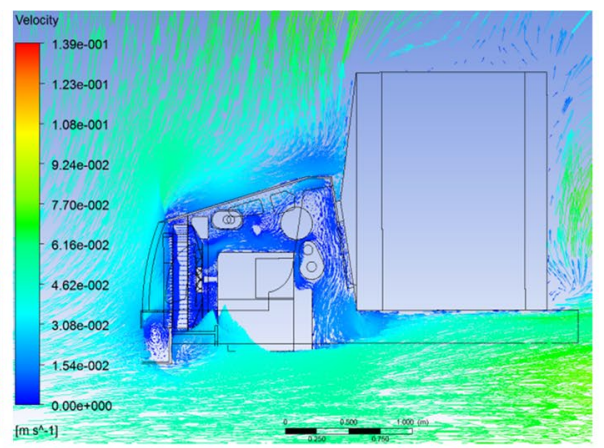

b Option 2

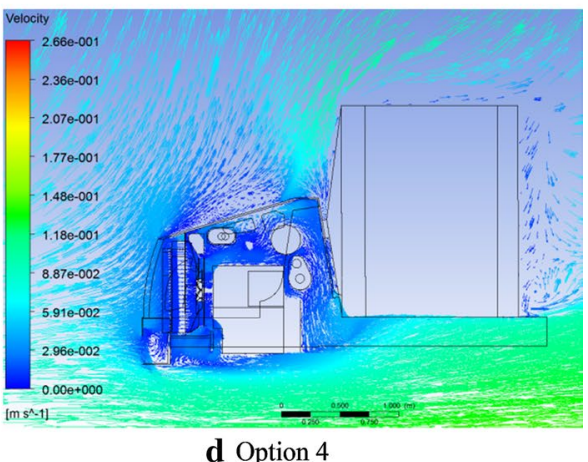

d Option 4

Fig. 5 Cross-sectional speed vector at idle $(x=0)$

increases, the intake air volume increases, the flow into the radiator group increases, and the fan pressure rises. The influence of the air hood on the cooling air volume is gradually reduced. Due to the compact structure of the heat-dissipating cabin and numerous components, gas flows through the heat-dissipating cabin toward the rear and the underside of the vehicle body, and is hindered by a plurality of components, resulting in separation and generation of complicated eddy currents, which also leads to an increase in internal flow resistance. In high-speed operating conditions, the vehicle speed is higher, the air velocity entering the air inlet is higher, the overall flow rate in the heat-dissipating cabin is higher, and the flow condition is better. It is more important to note that the fan pressure rise and air deflector are at idle speed and low speed in the loader-digger. Therefore, in order to increase the air intake flow rate under idle conditions and improve the fan efficiency, the influence of the position of the fan of the loader-digger on the flow performance in the radiating cabin is studied.

\subsection{Prediction of flow performance on the relative position of the fan and the radiator}

When the vehicle speed is low, the air flow in the cooling chamber is mainly generated by the suction action of the fan. Therefore, the relative position of the fan and the radiator set at idle is studied. Select the fan and radiator group relative positions are, respectively, 56, 76, 96, $116 \mathrm{~mm}$ to establish four kinds of programs, and radiator group and engine relative distance, inlet speed is 0 , the fan speed is $2200 \mathrm{rpm}$.

When the fan speed is $2200 \mathrm{rpm}$, the speed vector diagram of the four programs under idle conditions is shown in Fig. 5 It can be seen from the figure that the flow rate behind 


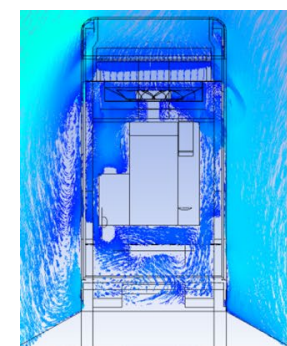

a Option 1

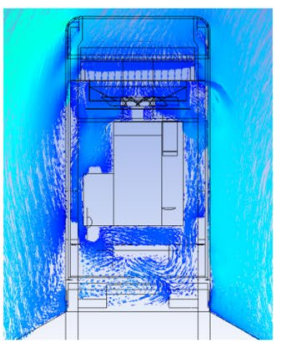

b Option 2

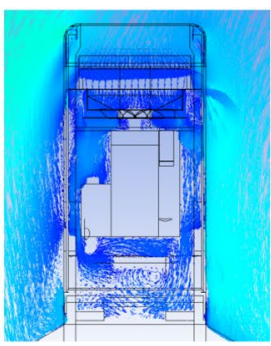

c Option 3

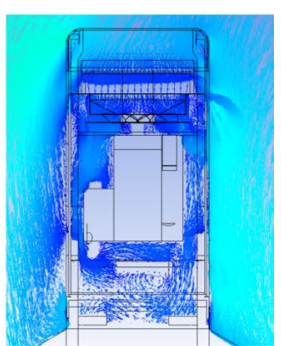

d Option 4

Fig. 6 Section speed vector at idle $(z=1.5)$

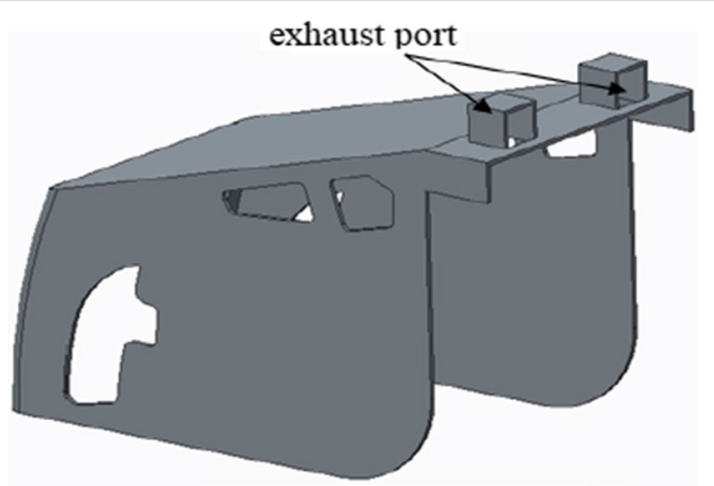

Fig. 7 Hood to increase the exhaust port

the engine of option 4 is low, and it is easy to cause waste heat to accumulate. The flow field in option 2 and option 3 for the radiating cabin is denser, which is conducive to the heat discharge from the radiating cabin.

The horizontal cross-section velocity vector at the center of the fan is shown in Fig. 6. It can be seen from the figure that the air flow path becomes smaller due to the smaller gap between the engine and the cab, thus making the airflow in the cab. There is certain stagnation and backflow between the engine and the engine, and the flow rate behind the engine is small, which is unfavorable to the heat dissipation performance of the engine and the entire radiating cabin. The vortex phenomenon occurs behind the mufflers of option 3 and option 4 and the vortex phenomenon behind the mufflers of option 1 and option 2 are weak.

\subsection{Optimization of the hood}

The hood acts as a shell to accumulate the high-temperature gas generated by the engine system, transmission system and hydraulic system in the heat dissipation cabin. Besides, when the vehicle speed is high, the air flow in the radiator compartment is mainly driven by the air entering the air inlet. At this time, the overall flow rate in the overall radiator compartment is high. However, due to the compact structure of the cooling chamber and numerous components, the flow velocity above the engine is relatively low. Therefore, the hood is optimized and improved, and the exhaust port shown in Fig. 7 is added. The entrance speed is $42.5 \mathrm{~km} / \mathrm{h}$, and the fan speed is $2200 \mathrm{rpm}$. 


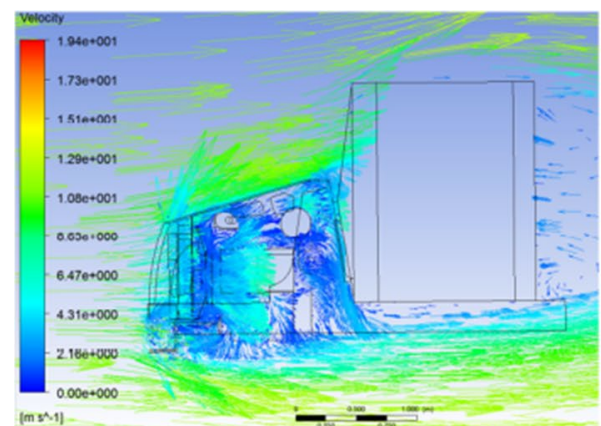

a After optimization

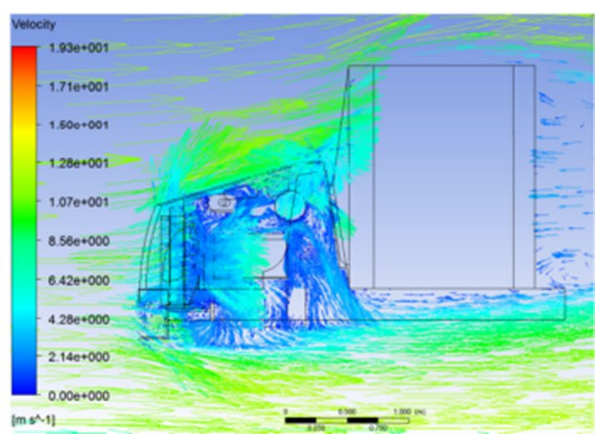

b Before optimization

Fig. 8 Cross-sectional speed vector before and after optimization $(x=0)$

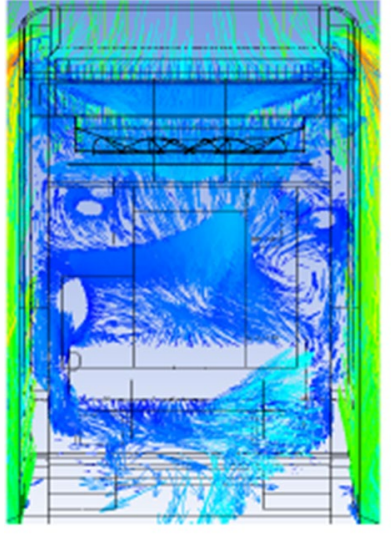

a Before Optimization

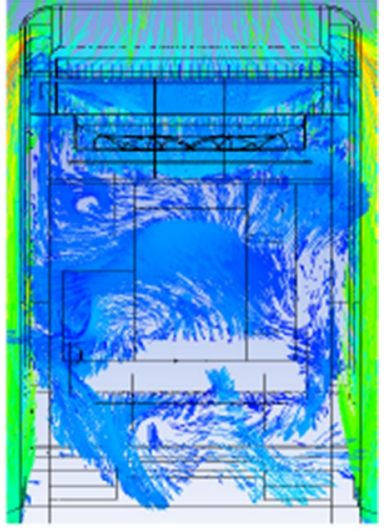

b After Optimization

Fig. 9 Cross-sectional speed vector before and after optimization ( $z=1.7)$

Figure 8 shows the vertical section speed vector at the center of the chiller cabin when the speed of the loader-digger is $42.5 \mathrm{~km} / \mathrm{h}$. Comparing the calculation results, it shows that after the optimization, the speed tends to increase significantly above the engine, and the flow velocity distribution behind the engine is more uniform.

Figure 9 shows the horizontal cross-section velocity vector at the center of the fan and at the center of the muffler. From the figure, it can be compared and found that the optimized flow field on the right side and rear side of the engine is more uniform than before the optimization. The phenomenon of eddy currents and stagnation becomes weaker. After optimization, the flow velocity above and behind the engine becomes faster, and the eddy current behind the muffler weakens, but a vortex is generated in front of the silencer due to air collision.

\subsection{Results of the simulation}

In this chapter, the radiator cabin of loader-digger is analyzed based on CFD. Firstly, the flow field in the radiator cabin at different speeds is simulated. It is concluded that the cooling fan and wind deflector have a greater impact under the condition of 
Table 2 Loader-digger parameters

\begin{tabular}{llll}
\hline Parameter & Value & Parameter & Value \\
\hline Operating hydraulic system pressure & $22 \mathrm{MPa}$ & Full load lift time on the loading side & $4.0 \mathrm{~s}$ \\
Engine power & $75 \mathrm{~kW}$ & Load down time at full load & $2.6 \mathrm{~s}$ \\
Maximum no-load engine speed & $2307 \mathrm{r} / \mathrm{min}$ & Load loading load unloading time & $1.6 \mathrm{~s}$ \\
No load machine quality & $890 \mathrm{~kg}$ & Action time three items and & $8.2 \mathrm{~s}$ \\
Full load machine quality & $10570 \mathrm{~kg}$ & I gear speed & $9.24 \mathrm{~km} / \mathrm{h}$ \\
Bucket rated capacity & $1 \mathrm{~m}^{3}$ & II gear speed & $16.21 \mathrm{~km} / \mathrm{h}$ \\
The bucket dug hard & $53.54 \mathrm{kN}$ & III gear speed & $27.4 \mathrm{~km} / \mathrm{h}$ \\
The moving arm lifts the force & $41.00 \mathrm{kN}$ & IV gear speed & $39.18 \mathrm{~km} / \mathrm{h}$ \\
\hline
\end{tabular}

loader-digger idling and low speed. Secondly, the relationship between the relative position of the cooling fan and the radiator and the fluidity in the cooling chamber is analyzed. For four different distance simulations, scheme 2 has the best overall performance. The optimal distance between the cooling fan and radiator is $76 \mathrm{~mm}$. Finally, two exhaust holes are added above the hood to improve the internal flow field performance of the radiator cabin.

\section{Heat balance test system based on wireless sensor network}

With the development of networks technology, wireless sensor network plays a key role in condition monitoring and data acquisition, and it has been widely used in industry [18-22]. In order to understand the working performance of excavator loader, the data collected by sensors are used to check whether the thermal balance performance meets the requirements under various load conditions, and the thermal balance state of the whole machine is analyzed. The vehicle heat balance of the 766A excavator loader under different working conditions was tested to evaluate the performance of the heat balance system.

\subsection{Heat balance test environment and machine configuration}

The test environment is fog free, rain free and snow free. The ambient temperature is more than $24{ }^{\circ} \mathrm{C}$, the air pressure is $95-102 \mathrm{kPa}$, and the wind speed is no more than $5 \mathrm{~km} / \mathrm{h}$. The test site shall be the site closest to the actual working condition (can be on the actual site or special test site).

The test prototype is the $766 \mathrm{~A}$ excavating loader produced by a certain manufacturer. The main parameters are shown in Table 2.

\subsection{Test instrument and data acquisition point arrangement}

During the operation of the loading excavator, various sensors are used to monitor various data of the heat balance system [23-26]. These data include video and pictures of the radiator, temperature parameters, etc.[27, 28]. Using sensors to obtain large amounts of data for condition assessment can help us adjust the heat balance system to achieve the best condition [29-32]. In order to analyze the heat balance of the excavator loader under various load conditions, the heat balance of the whole vehicle is tested, and the performance of the excavator loader system is evaluated. The test equipment 

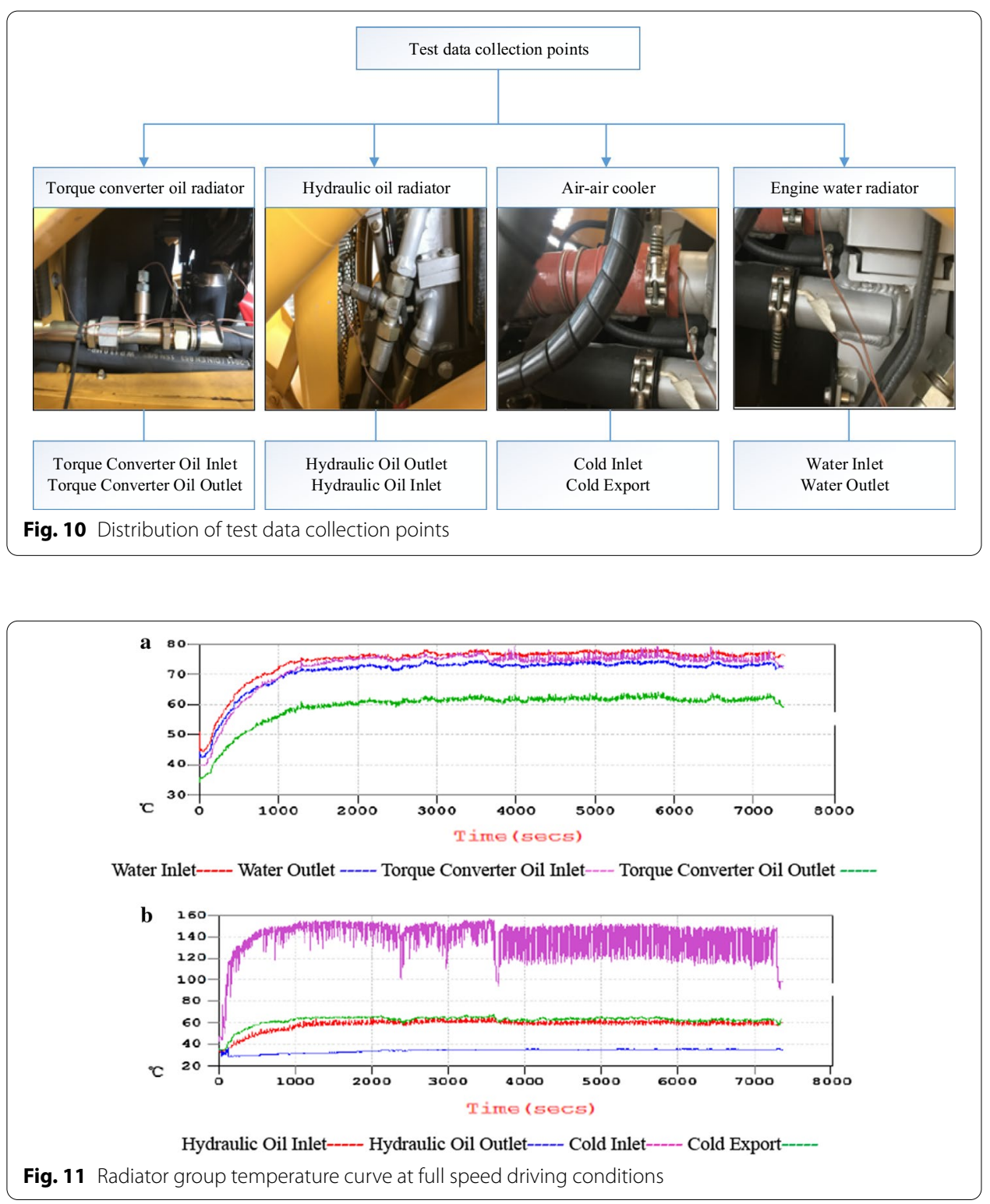

are arranged as follows: temperature measuring device (thermocouple wire) accuracy is $1{ }^{\circ} \mathrm{C}$, timer accuracy is $0.1 \mathrm{~s}$, data acquisition recorder, sampling frequency is $1 \mathrm{~Hz}$, Ncode instrument and Tce software. The test points are set to: engine cooling water inlet and outlet temperatures, hydraulic cooling oil inlet and outlet temperatures, converter cooling oil inlet and outlet temperatures, and air-to-air cooler inlet and outlet air temperatures, shown in Fig. 10.

\subsection{Analysis of test results}

The test condition is a sports car condition, that is, the highest-grade full-speed running condition. The test results are shown in Fig. 11. 
Table 3 Test results of full-speed driving conditions

\begin{tabular}{llll}
\hline Measured point & $\begin{array}{l}\text { Measured } \\
\text { temperature } \\
\left({ }^{\circ} \mathbf{C}\right)\end{array}$ & $\begin{array}{l}\text { Converted } \\
\text { temperature } \\
\left({ }^{\circ} \mathbf{C}\right)\end{array}$ & $\begin{array}{l}\text { Measured temperature } \\
\text { before improvement } \\
\left({ }^{\circ} \mathbf{C}\right)\end{array}$ \\
\hline Ambient temperature & 32 & 45 & 32 \\
Engine cooling water inlet temperature & 77 & 90 & 80 \\
Engine cooling water outlet temperature & 73 & 86 & 75 \\
Torque converter cooling oil inlet temperature & 76 & 89 & 82 \\
Torque converter cooling oil outlet temperature & 72 & 85 & 78 \\
Hydraulic cooling oil Inlet temperature & 62 & 75 & 63 \\
Hydraulic cooling oil outlet temperature & 35 & 48 & 35 \\
Air-air cooler inlet air temperature & 35 & - & 36 \\
Air-air cooler outlet air temperature & 153 & - & 160 \\
\hline
\end{tabular}

As can be seen from the figure, the maximum temperature of the heat balance system of the loader-digger is shown in Table 3. Since the loader-digger is often working under harsh conditions, the heat dissipation system temperature must be converted to the harshest environment, i.e., the ambient temperature is $45{ }^{\circ} \mathrm{C}$. Referring to Table 1 , it can be seen that the temperature of each radiator of the optimization scheme is lower than the temperature limit. The heat dissipation performance of the entire machine after optimization is up to standard. The optimized scheme can make the loader-digger operate in an efficient and energy-saving manner.

From the comparison between the actual measured temperature before and after optimization in Table 3, it can be seen that the overall temperature of the improved radiator group slightly decreases. Among them, the cooling effect of the air-air cooler and the torque converter oil radiator in the upper half of the radiator assembly is more significant than that of the engine radiator and the hydraulic oil radiator in the lower half, and the torque is converted after the temperature is converted. The oil cooler temperature is reduced by $6.3 \%$.

\section{Conclusions and outlooks}

The working environment of loader-digger is various, which requires high heat dissipation of the whole machine. Aiming at the problem that the temperature of some radiators in the heat dissipation system of loader-digger exceeds the standard, the CFD simulation is used to understand the effect of speed, relative distance between cooling fan and radiator and hood structure on the flow field in the radiator cabin. Then, the simulation results are verified by establishing a heat balance test system based on wireless sensor network. The test results show that the temperature of each radiator of the optimized scheme does not exceed the temperature limit, and the heat dissipation performance of the whole machine is good. The proposed scheme not only improves the cooling effect of the whole machine, but also dynamically adjusts the heat balance system.

Due to the limitation of some conditions, the research work in this paper is still limited. The contents to be improved and the further work to be carried out in this paper are as follows: 
1 Due to the complicated working conditions of stone shoveling and transporting by the excavator loader, it is necessary to analyze each movement in detail.

2 As the heat balance system is a dynamic cooling process, it needs to be combined with electromechanical fluid integration and dynamic simulation.

\section{Abbreviations}

CFX: A practical fluid engineering analysis tool; CFD: Computational fluid dynamics; MPV: Multi-purpose vehicle; FNM: Flow network modeling; LPG: Liquefied petroleum gas.

\section{Acknowledgements}

The authors acknowledged the anonymous reviewers and editors for their efforts in valuable comments and suggestions.

\section{Authors' contributions}

LL proposes the innovation ideas and theoretical analysis. CX conceived of the study, and participated in its design and coordination and helped to draft the manuscript. All authors read and approved the final manuscript.

\section{Funding}

No.

Availability of data and materials

Data sharing is not applicable to this article as no datasets were generated or analyzed during the current study.

\section{Competing interests}

The authors declare that they have no competing interests.

\section{Author details}

${ }^{1}$ Mechanical Engineering College, Jiangsu University of Science and Technology, Zhenjiang 212003, China. ${ }^{2}$ Jiangsu LiuGong Machinery Co., Ltd., Zhenjiang 212000, China.

Received: 17 May 2020 Accepted: 20 October 2020

Published online: 30 October 2020

\section{References}

1. J. Wang, C. Zhang, C. Zhang, et al., Influence of opening of passenger car rear door on heat dissipation of engine compartment. J. Agric. Mach. 43(9), 31-36 (2012)

2. F.C. Chang, S. Malipeddi, S. Uppuluri et al., Underhood thermal management of off-highway machines using 1D-network simulations. International Truck and Bus Meeting and Exhibition (2003)

3. M. Sortor, On-system engine fan measurement as a tool for optimizing heat balance system airflow performance and noise. SAE Int. J. Mater. Manuf. 4(1), 1221-1230 (2011)

4. D. Zhang, W. Wang, Application of CFD technology for thermal management of vehicle engine room. Dev. Innov. Mech. Electr. Prod. 25(6), 113-115 (2012)

5. M. Khaled, M. Ramadan, H. El-Hage et al., Review of underhood aerothermal management: towards vehicle simplified models. Appl. Therm. Eng. 73(1), 842-858 (2014)

6. M. Khaled, Fan air flow analysis and heat transfer enhancement of vehicle underhood heat balance systemtowards a new control approach for fuel consumption reduction. Appl. Energy 91(1), 439-450 (2012)

7. V. Damodaran, M. Rahman, Front-end cooling airflow performance prediction using vehicle system resistance. SAE International-Thermal Management SP1751, SAE Paper (2003)

8. K. Jahani, S. Beigmoradi, Under-hood air flow evaluation of pedestrian-friendly front-end style using CFD simulation. SAE Int. J. Passeng. Cars-Mech. Syst. 7(2), 787-792 (2014)

9. A.K. Saha, S. Acharya, Parametric study of unsteady flow and heat transfer in a pin-fin heat exchanger. Int. J. Heat Mass Transf. 46(20), 3815-3830 (2003)

10. C. Zhang, M. Uddin, X. Song et al., Simultaneous improvement of vehicle under-hood airflow and cooling drag using 3D CFD simulation. SAE Technical Paper (2016)

11. J.J. Qu, L.F. Li, T. Cui et al., Application of field synergy principle to analysis of flow field inunderhood of LPG bus. Comput. Fluids 103, 186-192 (2014)

12. J. Liu, B. Wang, S. Qin et al., Numerical simulation of heat dissipation performance of cooling module for construction vehicles with dual fan. J. Huazhong Univ. Sci. Technol. 46(4), 127-132 (2018)

13. X. Dong, X. Liu, Multi-objective optimal design of microchannel cooling heat sink using topology optimizationmethod. Numer. Heat Transf. Part A Appl. 77(1), 90-104 (2020)

14. X. Wang, H. Xu, J. Wang et al., Multi-objective optimization of discrete film hole arrangement on a high pressure turbine end-wall with conjugate heat transfer simulations. Int. J. Heat Fluid Flow 78, 108428 (2019) 
15. S. Lu, J. Zhang, R. Liang, C. Zhou, Refrigeration characteristics of a hybrid heat dissipation photovoltaic thermal heat pump under various ambient conditions on summer night. Renew. Energy 146, 2524-2534 (2020)

16. J.Wang, X. Liu, S. Chen et al., Reduced-scale model study on cable heat dissipation and airflow distribution of power cabins. Appl. Therm. Eng. 160, 114068 (2019). https://doi.org/10.1016/j.applthermaleng.2019.114068

17. C. Li, C. Zhang, M. Wu et al., Optimisation design of the inlet of an emergency power supply cabin based on its heat dissipation characteristics. Austr. J. Mech. Eng. (2019). https://doi.org/10.1080/14484846.2019.1678995

18. Y. Zeng, C.J. Sreenan, N. Xiong, L.T. Yang, J.H. Park, Connectivity and coverage maintenance in wireless sensor networks. J. Supercomput. 52(1), 23-46 (2010)

19. C. Lin, N. Xiong, J.H. Park, T. Kim, Dynamic power management in new architecture of wireless sensor networks. Int. J. Commun. Syst. 22(6), 671-693 (2009)

20. Y. Sang, H. Shen, Y. Tan, N. Xiong, Efficient protocols for privacy, matching, distributed, distributed datasets, in International Conference, Information, and Communications Security, pp. 210-227 (2006)

21. F. Long, N. Xiong, A.V. Vasilakos, L.T. Yang, F. Sun, A sustainable heuristic QoS routing algorithm for pervasive multilayered satellite wireless networks. Wirel. Netw. 16, 1657-1673 (2010)

22. W. Guo, N. Xiong, A.V. Vasilakos, G. Chen, C. Yu, Distributed k-connected fault-tolerant topology control algorithms with PSO in future autonomic sensor systems. Int. J. Sens. Netw. 12(1), 53-62 (2012)

23. Z. Huang, X. Xu, J. Ni, H. Zhu, C. Wang, Multimodal representation, learning for recommendation, IEEE Internet of Things. IEEE Internet Things J. 6(6), 10675-10685 (2019)

24. F. Hu, G. Wu, Distributed error correction EKF algorithm in multi-sensor fusion localization model. IEEE Access 8 , 93211-93218 (2020)

25. C. Lin, Y.X. He, N. Xiong, An energy-efficient dynamic power management in wireless sensor networks, in 20065 th International Symposium Parallel and Distributed Computing (2006)

26. Y. Liu, M. Ma, X. Liu, N. Xiong, A. Liu, Y. Zhu, Design and Analysis of Probing Route to Defense Sink-hole Attacks for Internet of Things Security. IEEE Trans. Netw. Sci. Eng. (2018). https://doi.org/10.1109/TNSE.2018.2881152

27. L. Dong, Q. Guo, W. Wu, Speech corpora subset selection based on time-continuous utterances features. J. Combin. Optim. 37(4), 1237-1248 (2019)

28. D. Jiang, G. Li, Y. Sun, J. Kong, B. Tao, Gesture recognition based on skeletonization algorithm CNN with ASL database. Multimedia Tools Appl. 78(21), 29953-29970 (2019)

29. Z. Wang, T. Li, N. Xiong, Y. Pan, A novel dynamic network data replication based on historical access and proactive deletion. J. Supercomput. 62(1), 227-250 (2012)

30. J. Li, N. Xiong, J.H. Park, C. Liu, M.A. Shihua, S.E. Cho, Intelligent design model of cluster supply chain with horizontal cooperation. J. Intell. Manuf. 23(4), 917-931 (2012)

31. Y. Yang, N. Xiong, N.Y. Chong et al., A decentralized and adaptive flocking algorithm for autonomous mobile robots, in The 3rd International Conference on Grid and Pervasive Computing (2008)

32. M. Zhang, D. Zhang, H. Yao et al., A probabilistic model of human error assessment for autonomous cargo ships focusing on human-autonomy collaboration. Saf. Sci. 130, 104838 (2020)

\section{Publisher's Note}

Springer Nature remains neutral with regard to jurisdictional claims in published maps and institutional affiliations.

\section{Submit your manuscript to a SpringerOpen ${ }^{\circ}$ journal and benefit from:}

- Convenient online submission

Rigorous peer review

- Open access: articles freely available online

- High visibility within the field

Retaining the copyright to your article

Submit your next manuscript at $\gg$ springeropen.com 